\title{
Diretrizes de Acessibilidade: Uma Abordagem Comparativa entre WCAG e e-MAG
}

\author{
Catharine F. Bach ${ }^{1}$, Simone Bacellar Leal Ferreira ${ }^{1}$, Denis S. Silveira ${ }^{2}$ e Ricardo \\ Rodrigues Nunes ${ }^{1}$ \\ ${ }^{1}$ Universidade Federal do Estado do Rio de Janeiro - UNIRIO \\ Av. Pasteur, 458, Urca-Cep: 22490040 - Rio de Janeiro, RJ, Brasil \\ ${ }^{2}$ Programa Engenharia de Produção COPPE/UFRJ \\ CT - Bloco: F, Sala: F113, CEP: 21941-972 - Rio de Janeiro/RJ, Brasil \\ catharine.bach@uniriotec.br, simone@uniriotec.br, denis@pep.ufrj.br , \\ ricardo.nunes@uniriotec.
}

\begin{abstract}
The present work presents a comparative study between two accessibility Standards: the international WCAG and the Brazilian e-MAG. In this study their main characteristics, similarities and differences are described. It was carried a research among Brazilian organizations in order to verify their adherence to the two models. Some tests were conducted for check the accessibility of the institutional sites. A questionnaire was prepared to analyze the adherence to guidelines. The results showed that both models are quite similar what indicates that the international one can fit the national necessities.
\end{abstract}

Resumo. O presente trabalho apresenta um estudo comparativo entre os padrões de acessibilidade: o internacional WCAG e o nacional e-MAG. Nesse estudo são descritas as principais características dos modelos, suas semelhanças e diferenças. Para isso, foi realizada uma pesquisa junto a organizações brasileiras de modo a verificar o grau de aderência aos dois padrões; foram conduzidos testes automatizados para checar a acessibilidade dos sites institucionais e foi elaborado um questionário para analisar o grau de aderência às diretrizes. Os resultados revelaram que as recomendações propostas pelos dois padrões apresentam poucas diferenças, indicando que o padrão internacional se adequa às necessidades nacionais.

\section{Introdução}

Acessibilidade é o termo geral usado para indicar a possibilidade de qualquer pessoa usufruir de todos os benefícios de uma vida em sociedade, entre eles, o uso da Internet [Nicholl 2001; ABNT 1994]. Essa definição, proposta inclusive pela Associação Brasileira de Normas Técnicas (ABNT), apesar de rigorosa, é fundamental, pois a acessibilidade só existe de fato quanto "todos" conseguem acesso a esses benefícios [Queiroz 2008].

A acessibilidade digital refere-se ao acesso aos recursos computacionais; a acessibilidade na Internet é o usufruto dos recursos da rede mundial de computadores e a acessibilidade na Web, ou e-acessibilidade, refere-se especificamente ao componente WWW (World Wide Web), ou Web [Acessibilidade Brasil 2008], [Sales e Cybis 2003]. 
Visando o acesso universal, no final dos anos 90, esforços começaram a ser desenvolvidos para promover a acessibilidade em aplicativos Web. Em 1999, foi lançada a primeira versão das Diretrizes para a Acessibilidade do Conteúdo da Web (WCAG 1.0), elaborada pelo grupo de trabalho do WAI (Web Accessibility Initiative) do comitê internacional W3C (World Wide Web Consortium), que regula os assuntos ligados à Internet. O WCAG 1.0 até hoje é uma referência de acessibilidade na Web.

Em dezembro de 2004 foi assinado, no Brasil, um decreto, regulamentando a acessibilização dos sites da administração pública, de interesse público ou financiado pelo governo. Para viabilizar a implantação dessa lei, foi criado um Comitê da ABNT que, após analisar diversas normas de acessibilidade de vários países desenvolveu o Modelo de Acessibilidade Brasileiro (e-MAG), elaborado pelo Departamento de Governo Eletrônico, para conduzir a acessibilização dos sites brasileiros [ENAP 2007; Sales e Cybis 2003].

O objetivo deste trabalho é o de apresentar um estudo comparativo entre os padrões de acessibilidade do W3C (WCAG) e do governo brasileiro (e-MAG), descrevendo as principais características encontradas nos dois modelos.

Para atingir este objetivo, inicialmente, foi feita uma pesquisa junto às organizações brasileiras afetadas pelo decreto a fim de verificar o grau de aderência dessas instituições aos dois padrões [Leal Ferreira 2007]. Para avaliar essa adesão, foram conduzidos testes automatizados para verificar a acessibilidade dos sites institucionais, através de um programa especialmente projetado para este fim; posteriormente foi elaborado um questionário on-line que permitiu que se analisasse melhor o grau de aderência às diretrizes de acessibilidade e por último foi elaborado um estudo comparativo entre os dois modelos. Um total de 87 organizações responderam ao questionário e, dentre essas, apenas 37 (42\%) conheciam o modelo brasileiro (e-MAG).

\section{Acessibilidade}

Acessibilidade é a possibilidade de qualquer pessoa usufruir todos os benefícios da vida em sociedade, entre eles o uso da Internet [ABNT 1994; Sales e Cybis 2003]. No entanto, como já mencionado, a acessibilidade digital é mais específica. Ela se refere apenas ao acesso aos recursos computacionais; a acessibilidade na Internet consiste na fruição dos recursos da rede mundial de computadores e a acessibilidade na Web, ou eacessibilidade, diz respeito especificamente ao componente da Web [Bos et al. 2008; Sales e Cybis 2003].

A acessibilidade na Web é a possibilidade de qualquer pessoa com alguma deficiência ou necessidade especial ter acesso aos seus recursos. Em outras palavras, não se trata apenas de acesso por deficientes. Logo, quando projetamos sistemas que serão executados no ambiente Web devemos nos preocupar com todos os tipos de usuários [Queiroz 2008]. Ou seja, não devemos esquecer que na Web encontramos todos os tipos de usuários e que a Web desempenha um papel importante no cotidiano de todas as pessoas, inclusive das que possuem necessidades especiais [Petrie et al. 2006].

\section{Diretrizes de Acessibilidade}

Devido a preocupação com o acesso universal, alguns governos passaram a implementar ou apoiar políticas de acessibilidade [Slatin e Rush 2003]. No entanto, 
existem diferentes diretrizes de acessibilidade propostas. Essa diversidade acarreta em uma falta de harmonia entre as diretrizes, tornando o trabalho de adequação aos diferentes modelos mais difícil para os profissionais envolvidos na construção de interfaces dos sistemas de informação que serão executados no ambiente Web [Tangarife e Mont'Alvão 2005].

Com o objetivo de tornar a Web acessível, o W3C (World Wide Web Consortium), consórcio voltado para o desenvolvimento de padrões Web criou, em 1999, o WAI (Web Accessibility Initiative), formado por grupos de trabalho voltados para a elaboração de diretrizes ligadas à garantia da acessibilidade do conteúdo na Web às pessoas com deficiência e às pessoas que acessam a rede em condições especiais de ambiente, equipamento, navegador e outras ferramentas Web [Henry 2008; ENAP 2007; Nevile 2005]. Os membros do W3C/WAI elaboraram as "Diretrizes de Acessibilidade de Conteúdo na Web” (WCAG 1.0), primeira versão das Diretrizes, lançada em maio de 1999, que permanece até hoje a principal referência de acessibilidade na Web [Henry 2008; Jacobs 2005].

No Brasil, a acessibilidade só começou fazer parte das políticas públicas a partir do ano 2000, com a promulgação das Leis Federais $n^{0} 10.048$ e $n^{0} 10.098$. A lei $n^{0} 10.048$, de 8 de novembro de 2000, trata do atendimento prioritário à acessibilidade das pessoas com deficiência aos meios de transporte e a outros serviços comunitários. Já a lei ${ }^{0} 10.098$, de 19 de dezembro de 2000, estabelece normas gerais e critérios básicos para a promoção da acessibilidade às pessoas com deficiência [ENAP 2007].

A expansão da Internet e a divulgação de informações e serviços do Governo na Internet, alinhados à atribuição do Governo de promover a inclusão social, levou à promoção de iniciativas que contribuíssem para a inclusão digital (parte da inclusão social). Nesse contexto, tornar os sites do governo acessíveis a toda população contribui para a inclusão digital e social. Em dezembro de 2004, foi assinado, no Brasil, o decreto $\mathrm{n}^{0}$ 5.296, regulamentando as leis anteriores e estabelecendo um prazo para a acessibilização de todo site da administração pública, de interesse público ou financiado pelo Governo. A fim de viabilizar a implantação dessa lei, criou-se um Comitê da ABNT incumbido de comparar as normas de acessibilidade de vários países e analisar as diretrizes propostas pelo W3C [Henry 2008]. Como resultado, desenvolveu-se o Modelo de Acessibilidade Brasileiro (e-MAG), elaborado pelo Departamento de Governo Eletrônico, com o propósito de facilitar e padronizar o processo de acessibilização dos sites [Acessibilidade Brasil 2008; ENAP 2007; Brasil 2005, 2005b; Sales e Cybis 2003].

\section{Web Content Accessibility Guidelines (WCAG)}

O WCAG 1.0, primeira versão das diretrizes, aprovado em 1999, é uma versão estável e até hoje referenciada [Henry 2008]. A segunda versão, o WCAG 2.0, até o presente momento, encontra-se em desenvolvimento. Por esse motivo, nesse trabalho é considerado o WCAG na sua versão 1.0.

No WCAG, através de suas diretrizes, é explicado como tornar o conteúdo da Web acessível para qualquer pessoa, inclusive para as pessoas com algum tipo de deficiência [Chisholm et al. 1999]. Ela possui quatorze recomendações ou diretrizes que são princípios gerais de um projeto acessível [Dias 2007; Chisholm et al. 1999]. 
Cada diretriz proposta possui pontos de verificação associados [Dias 2007; Chisholm et al. 1999]. Os pontos de verificação explicam como a recomendação aplica-se em uma área específica. Por exemplo, a diretriz "4. Indicar claramente qual o idioma utilizado" possui como um de seus pontos de verificação: "4.1 Identificar claramente quaisquer mudanças de idioma no texto de um documento, bem como nos equivalentes textuais (por ex., legendas)".

\subsection{Níveis de Prioridade do WCAG}

Cada ponto de verificação tem um nível de prioridade associado, embora existam exceções explicitamente indicadas nas diretrizes onde o nível de prioridade de um ponto de verificação pode mudar sob certas condições. O WCAG possui três níveis de prioridade [Dias 2007; Chisholm et al. 1999]:

- o nível de prioridade 1 está relacionado a pontos que o desenvolvedor deve, obrigatoriamente atender. Caso contrário, um ou mais grupos de usuários ficarão impossibilitados de acessar informações contidas nos documentos. Atender a esses pontos é requisito básico para que certos grupos acessem documentos disponíveis na Web;

- o nível de prioridade 2 está associado a pontos que o desenvolvedor deveria atender. Caso contrário, alguns grupos de usuários terão dificuldades em acessar informações dos documentos. Atender aos pontos desse nível remove barreiras significativas de acessibilidade;

- os pontos associados ao nível de prioridade 3 são aqueles que os desenvolvedores podem atender. Sem atender a esses pontos alguns grupos de usuários poderão encontrar dificuldades em acessar os documentos armazenados na Web. Atender a esses pontos aperfeiçoa o acesso ao site.

\subsection{Níveis de Conformidade do WCAG}

Sistemas em conformidade com as recomendações do WCAG 1.0 podem ser classificados de acordo com o nível de conformidade que atendem. Cada nível possui um símbolo de conformidade fornecido pelo W3C [Chisholm et al. 1999]. O nível de conformidade ' $\mathrm{A}$ ' indica que todas as recomendações de Prioridade 1 foram atendidas; o nível 'AA' indica que todas as recomendações de Prioridade 1 e 2 foram atendidas; já o nível 'AAA' indica que todas as recomendações de Prioridade 1, 2 e 3 foram atendidas.

Para identificar o nível de conformidade de um sistema, recomenda-se a utilização de ferramentas automáticas de avaliação de acessibilidade [Abou-Zahra et al. 2006], que verificam se a interface, páginas em HTML, está em conformidade com as diretrizes de acessibilidade.

\section{Modelo de acessibilidade do governo eletrônico (e-MAG)}

O Modelo de Acessibilidade de Governo Eletrônico (e-MAG) é composto por um conjunto de recomendações que devem ser consideradas no desenvolvimento e adaptação de conteúdos do Governo Brasileiro na Web. Toda instituição deve utilizá-lo ao construir e adaptar soluções de governo eletrônico, de modo que o processo de acessibilidade seja padronizado, de fácil implementação, adequado às necessidades 
brasileiras e em conformidade com padrões internacionais. Ele foi elaborado a partir de um estudo comparativo entre as normas de diversos países e o WCAG, sendo adaptado à realidade brasileira [Brasil 2005, 2005b]; é composto de duas visões: a Visão Técnica e a Visão do Cidadão.

A Visão do Cidadão, documento voltado ao público em geral, permite uma compreensão mais geral do Modelo com foco no cidadão [Brasil 2005b]. A Visão Técnica do Modelo é voltada aos profissionais que atuam no desenvolvimento. Essa visão apresenta as diretrizes e recomendações de acessibilidade, com informações técnicas que auxiliam o projetista na concepção das interfaces. Nessa Visão também são descritas as etapas do Processo de Acessibilidade. O e-MAG possui oito diretrizes e cada diretriz tem um conjunto de recomendações [Brasil 2005].

Para garantir a acessibilização de acordo com prioridades, o e-MAG adotou os mesmos três níveis de prioridade das recomendações estabelecidas pelo WCAG de forma evolutiva, preocupando-se com as recomendações de nível 1 antes das recomendações de nível 2 e 3. Depois, analisa-se e implementa-se recomendações de nível 2 e posteriormente as recomendações de nível 3 [Brasil 2005].

Assim como no WCAG 1.0, um sistema em conformidade com todas as recomendações de nível de prioridade 1 está classificado no nível de acessibilidade 'A'; a conformidade aos níveis de prioridade 1 e 2 classifica o sistema no nível de acessibilidade 'AA'; a conformidade aos níveis de prioridade 1,2 e 3 classifica o sistema no nível de acessibilidade 'AAA' [Brasil 2005, 2005b].

\section{Metodologia}

O estudo, de caráter exploratório, teve quatro etapas: (a) pesquisa bibliográfica e documental; (b) avaliação automática da adesão dos sistemas da administração pública ao e-MAG e ao WCAG 1.0; (c) estudo do processo de acessibilização nas organizações brasileiras afetadas pelo decreto; (d) estudo comparativo detalhado entre o e-MAG e o WCAG 1.0.

\subsection{Pesquisa Bibliográfica e Documental}

No primeiro momento, buscou-se compreender o princípio de acessibilidade e suas implicações. Durante essa etapa, algumas instituições forneceram softwares destinados a usuários deficientes visuais, que foram usados para navegar em jornais, sistemas de busca, e aprofundar a observação e análise dos aspectos levantados na literatura. Entre as instituições consultadas, encontram-se o IBC (Instituto Benjamin Constant http://www.ibc.gov.br/) e a UMIC (Unidade de Missão, Inovação e Conhecimento http://www.acesso.umic.pt/) da Agência para a Sociedade do Conhecimento do Governo de Portugal, que forneceu um kit de acessibilidade com softwares e artigos relacionados ao tema.

\subsection{Avaliação Automática da Adesão dos Sites da Administração Pública ao e- MAG e ao WCAG 1.0}

Para avaliar a adesão dos principais sistemas das esferas federal, estadual e municipal do governo brasileiro aos padrões de acessibilidade tanto do W3C (WCAG) quanto do governo brasileiro (e-MAG), foram conduzidos testes automatizados. Para viabilizar 
esses testes, foi construído um programa testador como a finalidade de submeter automaticamente cada interface à análise do avaliador daSilva [Da Silva 2008], especializado em validar a acessibilidade de sites. Esse programa foi desenvolvido pela empresa Holden Comunicação e foi denominado "Testador de Acessiblidade via daSilva”, com a linguagem PHP e usando o banco de dados MySQL [Holden Comunicação 2007].

Foram conduzidos três testes, realizados em momentos diferentes: um mês antes do decreto 5296 entrar em vigor (novembro de 2005), quatro meses após o término do prazo determinado pela legislação (março de 2006) e quatro meses após o prazo final da prorrogação do decreto, ou seja, um ano após o segundo teste (março de 2007). Esses testes serviram como um indicador que mostrava como os sistemas avaliados se comportavam com relação aos dois padrões (e-MAG e WCAG), objetos de estudo da presente pesquisa.

\subsection{Estudo do Processo de Acessibilização nas Organizações Afetadas pela Lei}

A terceira etapa teve por finalidade avaliar como as organizações públicas estão conduzindo (ou não) a acessibilização de seus sistemas e verificar o grau de aderência às diretrizes de acessibilidade. Para essa avaliação foi elaborado um questionário que ficou disponível na Internet entre os dias 26/03/2007 e 20/04/2007.

Para facilitar o usuário em sua tarefa de responder, o questionário foi projetado contendo nove perguntas fechadas, no formato múltipla escolha e com a característica de ser sensível ao contexto, ou seja, ele se expandia de acordo com as respostas a determinadas questões.

Para facilitar a análise dos dados, o questionário foi desenvolvido com um recurso que permitia contabilizar os resultados sendo que, a cada nova resposta, automaticamente a tabela de resultados era atualizada; para garantir o sigilo prometido aos respondentes, a tabela foi protegida por senha [Holden Comunicação 2007].

Foi enviado um e-mail explicativo para as principais organizações afetadas pelo decreto contendo informações sobre a pesquisa e a URL onde o questionário estava hospedado e, solicitando que o responsável respondesse às perguntas. $\mathrm{O} e$-mail do destinatário foi obtido acessando o URL de cada organização, seguindo a seguinte ordem: $e$-mail do departamento de informática, se disponível, seguido do “fale conosco" da organização, $e$-mail da ouvidoria da presidência e por fim da assessoria de comunicação. Dado o método adotado para o envio de e-mails, é difícil dimensionar a quantidade de mensagens que foram enviadas com o pedido de preenchimento do questionário. As respostas individuais de cada organização, bem como a listas das empresas que participaram da pesquisa são confidenciais.

\subsection{Estudo comparativo detalhado entre o e-MAG e o WCAG 1.0}

O estudo detalhado do e-MAG e do WCAG 1.0 teve como objetivo identificar as semelhanças e diferenças entre esses dois padrões de acessibilidade. O WCAG foi estudado por ser um padrão definido por um consórcio internacional, o W3C. Já o eMAG foi estudado por impactar diretamente as organizações afetadas pelo decreto de 2004. 
O estudo comparativo consistiu em analisar as recomendações propostas pelos dois modelos, identificar os níveis de prioridade das recomendações e quais os critérios utilizados para analisar a conformidade de uma interface às diretrizes propostas.

\section{Limitações}

Uma limitação da pesquisa foi a dificuldade para se conseguir contatar os responsáveis pela administração dos sistemas; apesar de ter sido garantido o sigilo dos resultados, muitos responderam que dependiam de uma autorização de seus superiores para participar da pesquisa e que não poderiam responder.

Um fator que em alguns momentos atrasou o andamento da pesquisa foi decorrente da forma que o avaliador daSilva exibe os resultados de sua análise; o daSilva não fornece os resultados em um formato interpretável pelo computador (XML, por exemplo). Com isso, a solução adotada pelo programa "Testador de acessibilidade via daSilva" para armazenar os resultados foi criar uma expressão regular (script) que obtém o número de erros e avisos a partir do código HTML gerado pelo daSilva. A principal desvantagem desse script é que, se o daSilva alterar a forma como os dados são apresentados, o sistema pára de funcionar corretamente até que a expressão regular seja atualizada. Para agravar, o software tinha que esperar um minuto entre cada consulta, o que, acrescido ao fato de cada sistema precisar de duas consultas (uma para WCAG e uma para EGOV (e-MAG)), tornou a etapa de “Avaliação dos Sistemas” muito lenta. No terceiro teste realizado (março de 2007), ocorreram três interrupções porque o daSilva interrompia por algum tempo a avaliação de acessibilidade realizada automaticamente com o "Testador de acessibilidade via daSilva".

\section{Análise dos Resultados}

\subsection{Análise dos Resultados Obtidos Durante a Avaliação Automática dos Sites}

Um grupo de 351 URL da administração pública ou de interesse público foi submetido em três momentos diferentes ao teste de adequação às diretrizes de acessibilidade do W3C (WCAG) e do governo brasileiro (e-MAG).

Nos testes conduzidos no início de novembro de 2005, um mês antes do prazo oficial para a acessibilização dos URL da administração pública determinado pelo decreto de 2004, apenas cinco dos 351 receberam conceito 'AAA' em acessibilidade pelos padrões WCAG e quatro pelos padrões e-MAG. Do número total, 43 cometeram cinco erros ou menos em WCAG e 48 cometeram cinco erros ou menos em e-MAG. Os demais cometeram mais de cinco erros no total. Nos testes conduzidos em março de 2006, quatro meses após terminado o prazo inicial oficial, apenas um obteve conceito 'AAA' tanto em WCAG quanto em e-MAG. Nenhum obteve conceito 'AAA' em apenas um dos dois padrões. Dos 351 testados, 89 cometeram cinco erros ou menos em WCAG e 95 cometeram cinco erros ou menos em e-MAG. Já no terceiro teste, conduzidos em março de 2007, quatro meses após o fim do prazo da prorrogação do decreto, ou seja, um ano após o segundo teste (março de 2007), 22 já estavam em conformidade com WCAG e 23 com o e-MAG. Nesse último teste 93 cometeram cinco erros ou menos em WCAG e 97 cometeram cinco erros ou menos em e-MAG. 


\subsection{Análise dos Resultados Obtidos Durante o Estudo do Processo de Acessibilização nas Organizações Afetadas pela Lei}

O questionário foi elaborado com quatro perguntas, cujos resultados foram sintetizados no formato de três tabelas (Tabela 1, 2 e 3). A análise das respostas enviadas pela empresas permitiu as seguintes considerações:

Tabela 1. Resultado comparativo do conhecimento sobre o decreto

\begin{tabular}{|c|c|c|c|c|c|}
\hline \multirow{2}{*}{ Empresa } & \multirow{2}{*}{ Total } & \multirow{2}{*}{ Percentual } & \multicolumn{3}{|c|}{ Sobre o Decreto } \\
\cline { 5 - 6 } & & & Conhecia & Não conhecia & $\begin{array}{c}\text { Não soube } \\
\text { responder }\end{array}$ \\
\hline Já adaptado & 19 & $22 \%$ & 19 & & \\
\hline Em adaptação & 27 & $31 \%$ & 26 & & 1 \\
\hline Não adaptado & 41 & $47 \%$ & 15 & 19 & 7 \\
\hline Total & 87 & & $60(69 \%)$ & $19(22 \%)$ & $8(9 \%)$ \\
\hline
\end{tabular}

\subsubsection{Pouca adesão às diretrizes de acessibilidade}

As Tabelas 1, 2 e 3 mostram uma visão geral de como as empresas estão com relação à acessibilização. Como se pode observar, dos 87 sistemas das esferas federal, estadual e municipal do governo brasileiro, ou de interesse público, que responderam ao questionário, apenas 19 (22\%) estão em total conformidade com as diretrizes de acessibilidade, 27 (31\%) estão com seus sistemas em processo de acessibilização e 41 (47\%) não estão acessibilizados. Esses dados são indícios de que é necessário conscientizar e divulgar para as organizações o papel fundamental que a Web representa no cotidiano das pessoas com necessidades especiais para que elas se empenhem em reunirem todos os esforços para acessibilização de seus sistemas.

\subsubsection{Pouco conhecimento sobre a legislação e prazos}

Pelas Tabelas 1 e 2 pode-se ver que 19 (22\%) organizações consultadas desconheciam o decreto 5296 e 41 (48\%) não sabiam que o prazo para acessibilizar os seus sistemas havia se encerrado. Trata-se de um valor elevado, uma vez que se tratam de organizações governamentais ou de interesse público, todas afetadas pelo decreto; esse desconhecimento mostra o quão relevantes e necessários são os estudos sobre o tema.

Tabela 2. Resultado comparativo do conhecimento sobre o prazo do decreto

\begin{tabular}{|c|c|c|c|c|}
\hline \multirow{2}{*}{ Empresa } & \multirow{2}{*}{ Total } & \multirow{2}{*}{ Percentual } & \multicolumn{2}{|c|}{ Sobre o Prazo } \\
\cline { 4 - 5 } & & & Conhecia & Não conhecia \\
\hline Já adaptado & 19 & $22 \%$ & 18 & 1 \\
\hline Em adaptação & 27 & $31 \%$ & 23 & 4 \\
\hline Não adaptado & 41 & $47 \%$ & 5 & 36 \\
\hline Total & 87 & & $46(53 \%)$ & $41(48 \%)$ \\
\hline
\end{tabular}


Tabela 3. Resultado comparativo do conhecimento sobre o e-MAG

\begin{tabular}{|c|c|c|c|c|}
\hline \multirow{2}{*}{ Empresa } & \multirow{2}{*}{ Total } & \multirow{2}{*}{ Percentual } & \multicolumn{2}{|c|}{ Sobre o e-MAG } \\
\cline { 4 - 5 } & & & Conhecia & Não conhecia \\
\hline Já adaptado & 19 & $22 \%$ & 14 & 5 \\
\hline Em adaptação & 27 & $31 \%$ & 18 & 9 \\
\hline Não adaptado & 41 & $47 \%$ & 5 & 36 \\
\hline Total & 87 & & $37(42 \%)$ & 50 (48) \\
\hline
\end{tabular}

\subsection{Análise dos Resultados Obtidos Durante Estudo Comparativo Detalhado entre o e-MAG e o WCAG}

Os resultados desse estudo revelaram que as recomendações propostas pelo e-MAG e pelo WCAG apresentam muitos pontos em comum, com algumas poucas recomendações do WCAG não encontradas no e-MAG. Tanto o WCAG quanto o eMAG utilizam três níveis de prioridade para classificar as recomendações, porém existem diferenças na classificação de recomendações equivalentes no e-MAG e no WCAG. Já a identificação de conformidade com as diretrizes utiliza o mesmo critério no e-MAG e no WCAG.

Uma outra diferença que deve ser seriamente considerada é que foram encontradas inúmeras ferramentas automáticas de avaliação de acessibilidade para o modelo WCAG, como o WebXact, o Lift, o Hera, Cynthia Says entre outras disponíveis [Henry 2008], enquanto para o e-MAG foi encontrado somente o validador DaSilva [Da Silva 2008]. Isso é uma limitação do e-MAG que não pode ser negligenciada, pois limita a avaliação em apenas um avaliador, enquanto o WAI recomenda a avaliação por pelo menos dois validadores [Henry 2008].

\subsubsection{Pontos de Verificação}

Baseado nos pontos de verificação do WCAG e nas recomendações do e-MAG, é possível identificar quais os pontos de verificação do WCAG correspondem às recomendações do e-MAG. No entanto, para verificar estas correspondências realizamos alguns experimentos com sites ligados ao governo. São eles: Governo Eletrônico, Instituto Benjamin Constant (IBC) e o Portal do Governo Brasileiro. A seguir apresentamos os resultados com estes experimentos, onde observamos muitos pontos em comum entre as duas diretrizes.

1. O site do Governo Eletrônico (http://www.governoeletronico.gov.br/):

- WCAG 1.0

a. 10 erros de prioridade 2, sendo: uma ocorrência do ponto de verificação 3.5; cinco ocorrências do 3.4 e quatro ocorrências do 12.4 .

b. 1 de prioridade 3, sendo uma ocorrência do ponto de verificação 10.4 .

- e-MAG 
a. 11 erros de prioridade 2, sendo: cinco ocorrências do 2.2 (que é semelhante ao 3.4 da prioridade 2 do WCAG); quatro ocorrências do 2.15 (que é semelhante ao 12.4 da prioridade 2 do WCAG); uma ocorrência do 2.10 (que é semelhante ao 3.5 da prioridade 2 do WCAG) e uma ocorrência do 2.14 (que é semelhante ao 10.4 da prioridade 3 do WCAG).

2. Instituto Benjamin Constant (http://www.ibc.gov.br/)

- WCAG 1.0

a. 3 erros de prioridade 2, sendo: uma ocorrência 3.5 e duas ocorrências do 3.4;

- e-MAG

a. 3 erros de prioridade 2, sendo: uma ocorrência 2.10 (que é semelhante ao 3.5 da prioridade 2 do WCAG); duas ocorrências do 2.2 (que é semelhante ao 3.4 da prioridade 2 do WCAG).

3. Portal do Governo Brasileiro (http://www.brasil.gov.br/)

- $\quad$ WCAG 1.0

a. 3 erros de prioridade 1 , sendo três ocorrências 1.1.

- e-MAG

a. 3 erros de prioridade 1 , sendo três ocorrências 1.11. (que é semelhante ao 1.1 da prioridade 1 do WCAG).

É interessante ressaltar que as avaliações tanto no WCAG como no e-MAG foram semelhantes, mudando apenas o número e o texto explicativo dos pontos de verificação.

\subsubsection{Níveis de Prioridade}

Apesar de existir uma correspondência entre a maioria das recomendações do E-MAG e os pontos de verificação do WCAG 1.0, o nível de prioridade dos itens correspondentes nem sempre é o mesmo.

No nível de prioridade 1 do e-MAG, existem diferenças no nível de prioridade estabelecido em relação a recomendações do WCAG 1.0. Essas diferenças são exibidas na Tabela 4.

Tabela 4. Resultado comparativo do nível de prioridade das recomendações do WCAG equivalentes às recomendações de prioridade 1 do e-MAG

\begin{tabular}{|c|c|}
\hline Prioridade no WCAG & Recomendação e-MAG (prioridade 1) \\
\hline 1,2 & 1.19 \\
\hline 2 & $1.10,1.16,1.18,1.19,1.23$ \\
\hline 3 & 1.1 \\
\hline 1,3 & $1.3,1.12$ \\
\hline
\end{tabular}




\begin{tabular}{|c|c|}
\hline 2,3 & 1.5 \\
\hline 3 & $1.8,1.13$ \\
\hline
\end{tabular}

No nível de prioridade 2 do e-MAG, as recomendações 2.9, 2.14 são equivalentes a recomendações classificadas como nível de prioridade 3 do WCAG 1.0.

No nível de prioridade 3 do e-MAG, as recomendações 3.1, 3.12, 3.13, 3.14 são equivalentes a recomendações classificadas como nível de prioridade 2 do WCAG 1.0.

O resultado do estudo comparativo pode indicar que ao promover a acessibilização de um site com base no WCAG, que é um padrão internacional, a acessibilização proposta pelo e-MAG seria também atendida. Assim, podemos considerar que o WCAG atende à realidade brasileira sem a necessidade de treinar os profissionais envolvidos no desenvolvimento de sites em mais de um padrão de acessibilidade.

\section{Considerações Finais}

Os resultados desse estudo mostram que, dentre as 87 organizações que responderam ao questionário, existe pouca adesão às diretrizes de acessibilidade, visto que $47 \%$ das organizações não estavam com seus sites acessibilizados. Outro problema encontrado foi que pouco se conhecia sobre o decreto de 2004 e o prazo estabelecido por ele.

O estudo comparativo entre o WCAG e o e-MAG permitiu identificar que as recomendações propostas pelos dois modelos apresentam poucas diferenças, indicando que o padrão internacional poderia ser utilizado para a realidade nacional. Logo, a utilização de um padrão como WCAG, que atende à realidade brasileira, evitaria a necessidade de treinar os profissionais envolvidos no desenvolvimento de sistemas em mais de um padrão de acessibilidade.

\section{Referências Bibliográficas}

ABNT - Associação Brasileira De Normas Técnicas. NBR 9050: acessibilidade de pessoas portadoras de deficiências a edificações, espaço, mobiliário e equipamento urbanos. Rio de Janeiro, 1994.

Abou-Zahra, S., et al (Ed.). Evaluating Web Sites for Accessibility: Overview, 2006: <http://www.w3.org/WAI/eval/Overview.html>. Acesso: 17/01/ 2007.

Acessibilidade Brasil. Recursos de acessibilidade. <http://www.acessobrasil.org.br/>. Acesso: 20/04/2008.

Bos, B., Çelik, T., Hickson, I., Lie, H. W. Aural style sheets. Cascading Style Sheets Level 2 Revision 1 Specification, 2007: <www.w3.org/TR/CSS21/aural.html>. Acesso: 16/02/ 2008.

Brasil, 2005. Recomendações de Acessibilidade para Construção e Adaptação de Conteúdos do Governo Brasileiro na Internet: eMag, Acessibilidade de Governo Eletrônico. Cartilha Técnica. Departamento de Governo Eletrônico. Secretaria de Logística e Tecnologia da Informação. Ministério do Planejamento, Orçamento e Gestão. Versão 2.0. 14 de Dezembro de 2005. $<$ https://www.governoeletronico.gov.br/acoes-e-projetos/e-MAG>. Acesso: 15/04/2008. 
Brasil, 2005b. Recomendações de Acessibilidade para Construção e Adaptação de Conteúdos do Governo Brasileiro na Internet: eMag, Acessibilidade de Governo Eletrônico. Modelo de Acessibilidade. Departamento de Governo Eletrônico. Ministério do Planejamento, Orçamento e Gestão. Versão 2.0. < https://www.governoeletronico.gov.br/acoes-e-projetos/e-MAG>.

Acesso: 15/04/2008.

Chisholm, W., Vanderheiden, G., Jacobs, I. (Ed.). Web Content Accessibility Guidelines 1.0. World Wide Web Consortium-1999: <http://www.w3.org/TR/WAIWEBCONTENT/>. Acesso: 13/01/2008.

Da Silva. O Primeiro Avaliador De Acessibilidade em Português para Websites.: $<$ http://www.dasilva.org.br/>. Acesso: 18/04/2008.

Dias, C. Usabilidade na Web: criando portais mais acessíveis. $2^{\text {a }}$ edição, Rio de Janeiro, Alta Books, 2007.

ENAP - Escola Nacional De Administração Pública - e-Mag - modelo de acessibilidade de governo eletrônico. Brasília, Curso a distância, 2007.

Henry, S.L. (Ed.) Web Content Accessibility Guidelines (WCAG) Overview. WWW Consortium 2008.< http://www.w3.org/WAI/intro/wcag.php>. Acesso: 20/03/ 2008.

Holden Comunicação. < http://www.holdencomunicacao.com/>.Acesso:5/1/2007

Jacobs, I. (Ed.) About the World Wide Web Consortium. World Wide Web Consortium, 2005. < http://www.w3.org/Consortium/>. Acesso: 11/03/2008.

Leal Ferreira, Simone Bacellar; SANTOS, R. C. ; SILVEIRA, Denis S . Panorama da Acessibilidade na Web Brasileira. In: ENANPAD Encontro Nacional dos Programas de Pós Graduação em Administração, 2007, Rio de Janeiro. Anais do ENANPAD 2007 Encontro Nacional dos Programas de Pós Graduação em Administração, 2007.

Nevile, L. Adaptability and accessibility: a new framework. In: Proc. Conference Of The Computer-Human Interaction Special Interest Group (Chisig) Of Australia On Computer-Human Interaction (Acm International Conference Proceeding Series), 19, 2005, ACM, 2005. v. 122. p. 1-10.

Nicholl, A. R. J. O ambiente que promove a inclusão: conceitos de acessibilidade e usabilidade. Revista Assentamentos Humanos, Marília, v.3, n. 2, p. 49-60, dez. 2001.

Petrie, H. et al. Remote usability evaluations with disabled people. In: Proc. SIGCHI., 2006. ACM, 2006. p. 1.133-41.

Queiroz, M. A. Bengala legal. <http://www.bengalalegal.com/>. Acesso: 2/04/2008.

Sales, M. B.; Cybis, W. A. Development of a checklist for the evaluation of the web accessibility for the aged users In: Proc. Latin American Conference On HumanComputer Interaction, ACM, 2003. v. 46. p. 125-33.

Slatin, J.M., Rush, S. Maximum Accessibility: Making Your Web Site Usable for Everyone. Addison-Wesley, 2003.

Tangarife, T., Mont’Alvão, C. “Estudo Comparativo Utilizando uma Ferramenta de Avaliação de Acessibilidade para a Web”.In:Proc.CLIHC '05,ACM,2005.pp.313318. 